\title{
Correlação entre a funcionalidade e a força muscular periférica em pacientes renais crônicos submetidos ò hemodiólise
}

\section{Correlation between functionality and a peripheral muscle strength in chronic renal patients undergoing hemodialysis}

\section{Bianca Pereira Costa ${ }^{1}$ \\ Isalina Gabriela de Almeida Gomes² \\ Larissa de Holanda Lessa ${ }^{3}$ \\ Dandhara Henrique de Farias ${ }^{4}$ \\ Ana Carolina do Nascimento Calles ${ }^{5}$ \\ Adoniran Rodrigues Farias ${ }^{6}$}

Endereço para Correspondência:

Larissa de Holanda Lessa

Residencial Jardim Europa, Od. F, lote 01, sn, Antares

57048-148 - Maceió, AL [Brasil]

lariissaholanda@hotmail.com

\footnotetext{
1 Clínica escola de Fisioterapia, Centro Universitário Tiradentes - UNIT/AL. Maceió, AL - Brasil. ORCID: https://orcid.org/0000-0002-8726-9664

2 Clínica escola de Fisioterapia, Centro Universitário Tiradentes - UNIT/AL. Maceió, AL - Brasil. ORCID: https://orcid.org/0000-0002-9772-5336

3 Departamento de Pós-Graduação, Faculdade Redentor. Maceió, AL - Brasil.

ORCID: https://orcid.org/0000-0003-3330-3350

4 Departamento de Pós-Graduação, Faculdade ESTÁCIO/FAL. Maceió, AL -Brasil.

ORCID: https://orcid.org/0000-0002-1761-7571

5 Coordenação adjunta de medicina, Centro Universitário Tiradentes - UNIT/AL. Maceió, AL - Brasil. ORCID: https://orcid.org/0000-0001-8035-5896

6 Centro de estudos, Hospital do Coração de Alagoas. Maceió, AL-Brasil.

ORCID: https://orcid.org/0000-0002-5524-3793
}

\begin{abstract}
Resumo
Introdução: A hemodiálise é um tratamento que acarreta diversas complicações nos sistemas muscular, metabólico e cardiorrespiratório, comprometendo a funcionalidade e força muscular periférica do paciente. Objetivo: Correlacionar à funcionalidade e a força muscular periférica em pacientes renais crônicos submetidos à hemodiálise. Métodos: Tratou-se de um estudo transversal com pacientes renais crônicos. Para avaliação de força muscular foi o utilizado o Medical Research Council (MRC). Durante a sessão de hemodiálise foi aplicado o questionário de Medida de Independência Funcional (MIF), que avalia a funcionalidade do indivíduo. Resultados: No estudo não foi encontrada correlação significativa entre MRC Total e MIF Total. Entretanto, foi observada uma correlação significativa e negativa entre idade e MRC total Conclusão: Foi possível analisar o perfil dos pacientes acometidos pela Doença Renal Crônica (DRC). Constatou-se que pacientes renais que apresentavam idade mais avançada possuíam um grau de força muscular reduzida.
\end{abstract}

Descritores: Doença renal crônica; Fisioterapia; Força Muscular.

\begin{abstract}
Hemodialysis is a treatment that entails several complications in the muscular, metabolic and cardiorespiratory systems, compromising the functionality and the peripheral muscular strength of the patient. Objective: To correlate peripheral muscle function and strength in chronic renal patients undergoing hemodialysis. Methods: This was a cross-sectional study with chronic renal patients. For evaluation of muscle strength was used the Medical Research Council (MRC). During the hemodialysis session, the Functional Independence Measure (MIF) questionnaire was applied, which evaluates the individual's functionality. Results: No significant correlation between MRC Total and MIF Total was found in the study. However, a significant and negative correlation was observed between age and total RRM. Conclusion: It was possible to analyze the profile of patients affected by chronic kidney disease (CKD). It was found that renal patients who were more advanced age had a reduced degree of muscular strength.
\end{abstract}

Keywords: Chronic Kidney Disease; Physiotherapy; Muscle Strength. 


\section{Introdução}

O número de pacientes portadores da doença renal crônica (DRC) vem aumentando progressivamente em todo mundo. Em 1994, o Brasil tinha 24.000 pacientes mantidos em programa de hemodiálise, em 2004, dados mundiais mostraram que os Estados Unidos, o Japão e o Brasil eram os três primeiros em número de pacientes com DRC, sendo está um grave problema de saúde pública ${ }^{1}$. Dados da Sociedade Brasileira de Nefrologia indicam que no Brasil há 91.314 pacientes em hemodiálise, com um aumento de $114,4 \%$ no número de casos de 2000 a $2011^{2}$.

A DRC é uma síndrome que inclui diversas nefropatias caracterizada por evolução lenta e progressiva, acarretando a diminuição da função renal, por resultado da destruição dos néfrons e incapacidade dos rins de realizar suas funções homeostáticas, apresentando ainda etiologia desconhecida, mas de acordo com alguns estudos está associada a fatores como a hipertensão arterial sistêmica e diabetes mellitus como as principais causas, além do tabagismo e dislipidemias ${ }^{3,4}$. Para Reboredo ${ }^{5}$ uma das maiores causas de mortalidade em pacientes com DRC é de origem cardiovascular, atingindo um índice anual em torno de aproximadamente, 9\%, ou seja, de 10 a 20 vezes maior se comparado à população geral.

A HD é uma terapia que consiste na circulação extracorpórea do sangue, onde o sangue vai fluir por um sistema de tubos para um dialisador, filtrando os resíduos tóxicos e excesso de líquido e em seguida, retoma para o organismo do paciente $^{6}$. O tratamento hemodialítico pode ocasionar diversas complicações que podem ser: metabólicas, cardiorrespiratórias e musculares. O sistema muscular é gravemente afetado pela deterioração musculoesquelética, atrofia por desuso e fraqueza muscular generalizada como resultado da patogenia da miopatia urêmica, que se conceitua como o acúmulo de toxinas no músculo que leva a perda progressiva das propriedades de força ${ }^{1}$.
Devido a essa perda de força, principalmente pela hipotrofia muscular das fibras tipo I e II, os pacientes com DRC apresentam uma capacidade aeróbica relativamente baixa, que leva ao rápido e progressivo descondicionamento físico, que pode estar associado também a descoloração da pele, emagrecimento, edema, fadiga, alterações pulmonares e consequentemente diminuição da funcionalidade do indivíduo ${ }^{7}$.

A capacidade funcional que está definida como a capacidade de realizar algo pelos próprios meios também ficará alterada, comprometendo as condições motoras e cognitivas, interferindo na vida dos pacientes e limitando as realizações das atividades de vidas diárias ${ }^{8}$.

Tendo em vista o achado na literatura pesquisada, pacientes com DRC submetidos à hemodiálise tendem a ter vários comprometimentos supracitados que reduzem a força muscular desses indivíduos, consequentemente podem afetar a funcionalidade, viu - se necessária uma investigação com o objetivo de correlacionar à funcionalidade e a força muscular periférica em pacientes renais crônicos submetidos à hemodiálise.

\section{Metodologia}

Realizou-se um estudo transversal no setor de hemodiálise da Unidade de Nefrologia de Alagoas - UNIRIM. O estudo foi aprovado pelo Comitê de Ética e Pesquisa do Centro Universitário Tiradentes - UNIT, MaceióAlagoas, parecer $\mathrm{n}^{\circ}$ 829.666, seguindo as diretrizes e normas vigentes regulamentadoras sobre pesquisa, envolvendo seres humanos, oriundas da Resolução 466/12 do Conselho Nacional de Saúde/Ministério da Saúde.

Para o cálculo do tamanho da amostra, foi utilizado o critério de conveniência com amostra não-probabilística, sendo avaliados durante os meses de outubro e novembro de 2014, 66 pacientes com diagnóstico clínico de doença renal crônica (DRC), que realizavam hemodiálise e maiores de 18 anos. Foram excluídos da amostra 
pacientes que apresentavam dificuldade no entendimento do comando verbal e pacientes amputados que não conseguiam realizar o teste de força muscular.

A coleta só foi iniciada após esclarecimentos dos objetivos do estudo, elucidação dos procedimentos que iriam ser realizados e pela assinatura dos participantes do Termo de Consentimento Livre e Esclarecido - TCLE. Inicialmente foi aplicado um questionário de avaliação inicial, contendo: identificação, idade, sexo e comorbidades apresentadas pelo doente.

Para avaliação de força muscular foi o utilizado Medical Research Council (MRC) antes de o paciente iniciar a sessão de hemodiálise, através de um único avaliador, para que não houvesse variação dos resultados da pesquisa. Durante a sessão de hemodiálise foi aplicado o questionário de Medida de Independência Funcional (MIF), que avalia a funcionalidade do indivíduo.

\section{Avaliação da funcionalidade}

$O$ instrumento para quantificar o grau de funcionalidade, de pacientes com DRC foi o questionário "Medida de Independência Funcional" (MIF), traduzido e validado no Brasil em 2000 por Riberto 9 . O questionário foi aplicado por um único pesquisador, sendo as perguntas e todas as opções de resposta lidas ao paciente. Estão descritos dois domínios na MIF, o motor onde as atividades avaliadas estão os autocuidados, transferências, locomoção, controle esfincteriano, e o cognitivo onde foram avaliadas a comunicação e cognição social, que inclui memória, interação social e resolução de problemas. Cada uma dessas atividades é avaliada e recebe um escore que parte de 1 (dependência total) a 7 (independência completa), assim a pontuação total varia de 18 a 126 . O valor 1 equivale à dependência total. Os níveis 2, 3 e 4 correspondem, respectivamente, à assistência máxima, moderada e mínima. O nível 5 diz respeito à supervisão, estímulo ou preparo, quando é necessária a presença, sugestão ou encora- jamento de outra pessoa, sem contato físico. O nível 6 se refere à independência modificada, quando as atividades precisam de uma ajuda técnica, adaptação, uma prótese realizada em tempo excessivo, e o nível 7 é a independência completa, as tarefas são realizadas sem ajuda e num tempo razoável ${ }^{7}$.

\section{Avaliação da força muscular periférica}

A avaliação do grau de força manual muscular foi verificada através do índice Medical Research Council - MRC - graduando a força muscular de 0 a 5, sendo grau 0: ausência de contração muscular, grau 1: contração muscular discreta, grau 2: movimento ativo no plano horizontal com a eliminação da gravidade, grau 3: movimento ativo contra a ação da gravidade, grau 4: movimento ativo com ação da gravidade e resistência, grau 5: força muscular normal, onde quanto maior for a pontuação maior o desempenho na atividade realizada nos grupos musculares selecionados. A avaliação da força muscular foi avaliada por um único pesquisador antes da hemodiálise, onde foram avaliados grupos de 6 músculos de cada lado (deltoide, bíceps braquial, extensor do punho, íleo-psoas, quadríceps e tibial anterior) com a resistência do pesquisador para cada movimento, sendo todos os movimentos explicados e demonstrados aos pacientes antes da sua realização. Após essa avaliação foi calculado o percentual de força através do escore do MRC. A pontuação total varia entre 0 (tetraparesia completa) a 60 (força muscular normal). Pontuação entre 48 e 37 pontos na escala do MRC são considerados portadores de fraqueza significativa; os que apresentam 36 pontos ou menos são classificados como severamente fracos ${ }^{10}$.

\section{Anớlise estatística}

Os dados estão apresentados como média e desvio-padrão ou como frequências absolutas 
e relativas. As relações entre as variáveis foram exploradas por meio de análises de correlação de Pearson e por meio de regressões lineares multivariadas, com o objetivo de descontar os efeitos da idade e do sexo sobre as relações. Para todas as análises foi utilizado um valor de alfa igual a 5\%, com auxílio do software Statistical Package for the Social Sciences v 20.0 (IBM Inc, Chicago, IL).

\section{Resultados}

Foram estudados 65 pacientes, sendo $56,9 \%$ (37) do gênero masculino e $43,1 \%$ (28) do gênero feminino, onde $76,9 \%$ apresentavam hipertensão arterial sistêmica e $40 \%$ da amostra apresentavam diabetes mellitus. A idade média total foi de $59,8 \pm 16,2$ anos, a menor e maior idade encontrada, na amostra foram 25 e 91 anos. A média do MRC total foi de $51,6 \pm 9,0$ e do MIF total foi de $119,6 \pm 15,5$, conforme demostrado na Tabela 1.

Tabela 1: Distribuição por média dos valores de idade e das varióveis MRC e MIF

\begin{tabular}{c|c|c}
\hline Variável & Média & Desvio Padrão \\
\hline Idade (anos) & 59,8 & 16,2 \\
\hline MRC Total & 51,6 & 9,0 \\
\hline MIF Total & 119,6 & 15,5 \\
\hline
\end{tabular}

MRC - Medida da força muscular periférica total; MIF - Medida da independência funcional total.

Fonte: dados da pesquisa.

Na pesquisa não foi encontrada correlação significativa entre MRC Total e MIF Total ( $\mathrm{r}=$ $0,175 ; \mathrm{p}=0,16)$, conforme demonstrado abaixo na figura 1, a amostra avaliada foram encontrados indivíduos tanto com valores normais para MRC total quanto para MIF total, e valores diminuídos para MRC com MIF total normal.

Foi encontrada na pesquisa uma correlação significativa e negativa entre idade e MRC total ( $\mathrm{r}=-0,506 ; \mathrm{p}<0,001)$, conforme descrito na figura 02 citada abaixo, ou seja, quanto maior a idade, menor a força muscular periférica. Logo

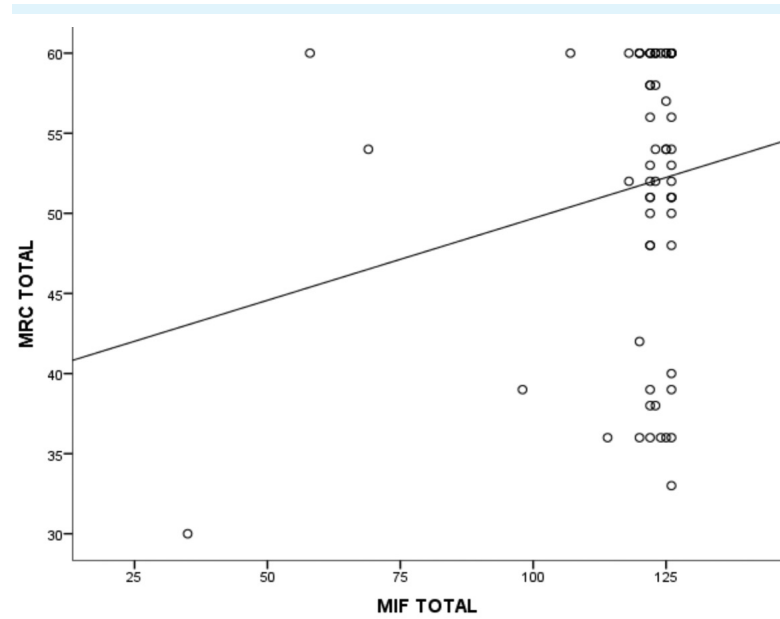

Figura 1: Correlação entre os valores do MRC Total x MIF TOTAL

Fonte: dados da pesquisa.

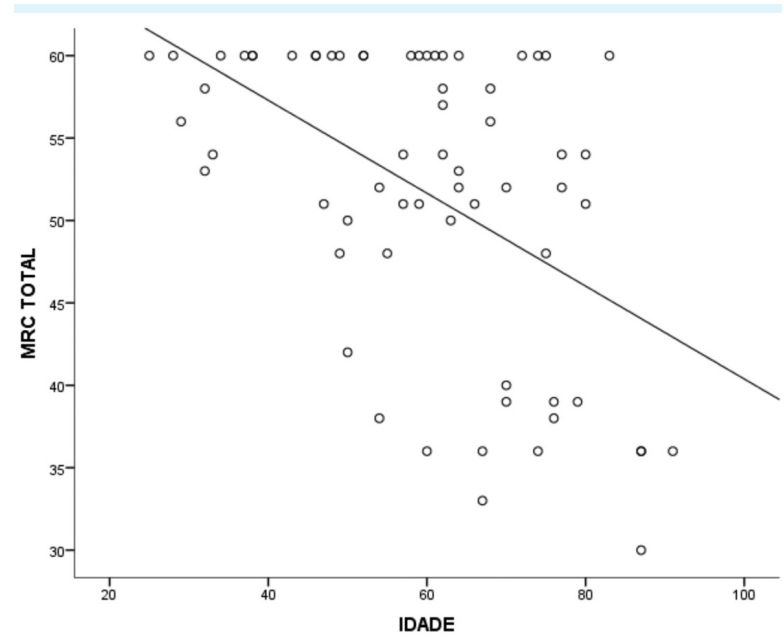

Figura 2: Correlação entre a idade e os valores do MRC total

Fonte: dados da pesquisa.

os pacientes que apresentaram diminuição da força muscular periférica são os pacientes que apresentavam maior faixa etária.

Em relação ao MIF total foi encontrada uma média de 119,6 indicando independência completa. Contudo, como demonstrado abaixo na figura 03, os subitens do MIF foram encontrados valores da média para MIF cognição social de 20,13 MIF, comunicação de 13,52, MIF locomoção 13,2, MIF mobilidade 20,2, MIF controle de esfíncter 13,47 e MIF autocuidado de 40,5 . 


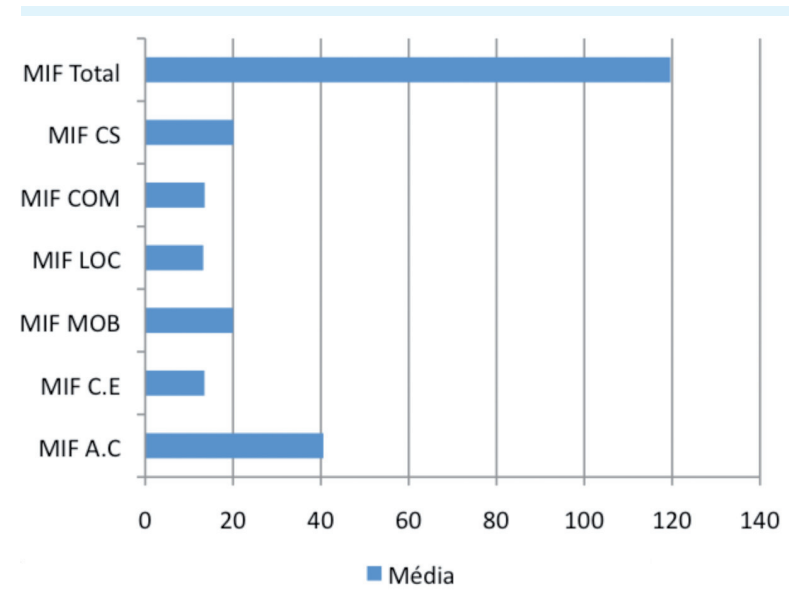

Figura 3: Distribuição mediana dos subtipos da MIF

Fonte: dados da pesquisa.

\section{Discussão}

De acordo com o censo realizado pela Sociedade Brasileira de Nefrologia (2010) indicam que a prevalência de pacientes renais crônicos é do gênero masculino atingindo um total de $67,7 \%$ dos $\operatorname{casos}^{11}$, no presente estudo confirmou-se esses dados com $56,9 \%$ sendo indivíduos homens.

A média da idade de pessoas acima de 60 anos da amostra do nosso estudo foi de 54,5\% da população estudada, dados encontrados também em outros estudos, em que a média de idade foi de $65,5 \pm 16,2$ anos, sendo $65,2 \%$ dos pacientes com idade superior a 60 anos $^{12}$.

Em relação aos fatores de risco, em nosso estudo $76,9 \%$ apresentavam-se com HAS e $40 \%$ com DM, que confirma com outros estudos de Bucuvic et al, 2011 onde 44,4\% apresentaram HAS e $61,9 \%$ DM e $52 \%$ tinham HAS, $26 \%$ para DM no estudo de Soares, 2011. Bortolotto ${ }^{13}$ diz que a HAS pode não causar a DRC, mas pode ser determinante para a progressão e agravamento da DRC.

Partindo dos dados estudados na pesquisa, a hemodiálise acarreta desequilíbrios em vários sistemas em longo prazo junto com a evolução da doença, ocasionando redução da capacidade funcional e da força no sistema musculoesque- lético ${ }^{14}$. Para Coelho ${ }^{15}$ a DRC traz consequências em vários sistemas, onde o musculoesquelético é gravemente afetado, apresentando déficit na funcionalidade e na força muscular do indivíduo acometido. Entretanto, foi evidenciado no presente estudo que não houve ligação direta entre as variáveis, encontrando valores de independência funcional normal e o de força muscular periférica diminuída.

Em nossa pesquisa, em relação ao escore do MIF total foi de 119,6 indicando independência completa dos pacientes acometidos com DRC, corroborando com estudos de Oller $^{8}$ com um escore de 118,38 pontos indicando também uma independência completa para funcionalidade.

De acordo com Côrrea ${ }^{16}$, em seu estudo avaliando a capacidade funcional através do TC6M, não foram encontrados valores significativos, indicando que os pacientes de sua amostra possuíam uma independência normal. Resultados semelhantes foram observados em nosso estudo, onde os indivíduos não apresentaram uma taxa de dependência funcional significativa, diferentemente do estudo de Reis ${ }^{17}$, encontrado na literatura, onde esses valores se encontraram abaixo do normal indicando que a capacidade funcional dos pacientes apresenta uma diminuição significativa.

A independência funcional e força muscular periférica dos pacientes estudados não demonstraram correlação com diferença significativa, além disso, muitos obtiveram valores próximos da normalidade, isso pode ser explicado pelo fato dos pacientes não apresentarem dificuldade na realização das atividades diárias, ao contrário do que se encontra em ouros estudos que a independência funcional se mostra reduzida em indivíduos com $\mathrm{DRC}^{18}$ e que a essa, pode não debilitar apenas a função renal, mas também provocar alterações físicas associadas ao tratamento, que constituem fatores limitantes das atividades diárias, tornando-se necessário um tratamento de reabilitação ${ }^{19}$, e em nosso estudo mesmo tendo uma média próxima ao valor normal para a força muscular periférica, 23, 07\% dos valores do teste MRC mostraram-se abaixo do normal. 
Em seu estudo Grassellii ${ }^{20}$, referiu-se ao funcionamento físico quanto ao grau em que a saúde física interfere no trabalho e em outras atividades diárias, e que leva a um rendimento menor que o desejado, porém, os dados de nossa pesquisa indicaram que tais fatores não afetaram negativamente a funcionalidade dos pacientes em tratamento hemodialítico, pois os valores obtidos sobre a medida de independência funcional não se mostraram significativos ao ponto de interferir na realização de atividades diárias, apresentando uma média de 119,6 pontos no MIF total. No estudo de Mariano ${ }^{19}$, a pontuação da média do MIF total foi 110 pontos, indicando uma dependência moderada, portanto não prejudicando as realizações das atividades de vidas diárias do paciente, corroborando com os dados da nossa pesquisa.

Em relação ao MRC total e idade foi encontrada na pesquisa uma correlação significativa e negativa $(\mathrm{p}<0,001)$ onde indica que quanto maior a idade, menor a força muscular periférica. As alterações encontradas nas fibras musculares são devidas não só pelo tratamento intradialítico, mas também, pela presença da miopatia urêmica, que ocasiona a atrofia e a fraqueza muscular, assim como pela deterioração normal da fisiologia humana do envelhecimento. A prevalência da IRC aumenta com a idade e aproximadamente $17 \%$ dos indivíduos maiores de 60 anos apresentam maior probabilidade de desenvolver a doença e uma acentuada perda da força muscular ${ }^{21}$.

Soares ${ }^{22}$, em seu estudo afirma que o paciente renal crônico apresenta excesso de líquidos corporais, o que deixam os órgãos congestos, inclusive os pulmões. Portanto a tolerância desses pacientes ao exercício fica gravemente reduzida, como afirma Costa ${ }^{23}$, que vários incômodos foram encontrados em pacientes com DRC durante a hemodiálise como dores musculares, câimbras, coceiras, fraqueza muscular, diminuição da ingestão de líquido, diminuição da ingestão alimentar e capacidade de trabalhar, o que afeta diretamente o desempenho físico desses pacientes em atividades diárias, diminuindo a independência funcional e a força muscular periférica. Esses achados não foram significativos em nossa pesquisa, pois nossa população estudada não apresentou acentuada perda de força muscular periférica e de funcionalidade.

Ainda discorrendo sobre funcionalidade, no estudo de Oller ${ }^{8}$ foi encontrada uma média de 118,38 pontos, indicando um nível de independência completa, média essa achada em nossa pesquisa com 119,6 pontos. Destaca-se para as dimensões da MIF motora, no autocuidado uma média de 40,56 pontos, onde se obteve um escore com maior pontuação e indicação de independência, e escores menores para a dimensão locomoção, valores achados também no estudo de Oller $^{24}$, onde foi observado um maior escore para auto cuidado, e menores escores para a dimensão locomoção, concordando com nosso estudo.

Segundo Kovelis ${ }^{25}$, a hemodiálise promove a degradação da musculatura assim como as proteínas de todo o organismo. A fraqueza muscular generalizada encontrada nos pacientes que realizam hemodiálise afeta predominantemente os membros inferiores e a musculatura proximal, esses dados explicam o fato de pessoas de nossa amostra com DRC apresentarem diminuição da força muscular periférica, principalmente se a idade desses indivíduos forem mais avançadas, porém, esses valores não se mostram diferentes de uma pessoa sem alteração da força muscular, o que indica que nossa amostra não apresenta tantos efeitos deletérios ocasionados pela hemodiálise.

Barreto $^{26}$, diz que em pessoas que realizam hemodiálise a musculatura se atrofia e como consequência, ocorre no organismo uma fraqueza generalizada, causada pela perda de força, que comparada a de indivíduos normais é de 30 a $40 \%$ menor, levando o paciente ao descondicionamento físico. Porém, em nosso estudo essa perda de força muscular não foi tão significativa, pois a maioria dos pacientes estudados apresentaram MRC > 48, o que não indica presença de fraqueza muscular.Segundo Schneider ${ }^{27}$, ocorrem alterações fisiológicas no indivíduo com 
DRC, como diminuição da massa muscular e da capacidade para o exercício, que podem ser provocadas pela inatividade física e pelo fato dessas pessoas terem várias complicações fisiológicas que contribuem para a inatividade física, tendo assim diminuições rápidas na sua capacidade funcional, como mostra nossa pesquisa que 95, $38 \%$ das pessoas estudadas apresentaram independência funcional modificada ou completa.

\section{Conclusão}

Diante da pesquisa foi possível analisar o perfil dos pacientes acometidos pela Doença Renal Crônica (DRC). Verificou-se que a Hemodiálise ocasiona efeitos deletérios nos sistemas muscular, metabólico e cardiorrespiratório dos indivíduos, o que acarreta uma grave fraqueza muscular e limitação na funcionalidade, entretanto, em nosso estudo não foi observado isso, pois os indivíduos apresentavam um grau de independência completa. Porém foi possível constatar que pacientes renais que apresentavam idade mais avançada possuíam um grau de força muscular reduzida.

\section{Referências}

1. Cury JL, Bunetto A F, AydoS, RD. Efeitos negativos da insuficiência renal crônica sobre a função pulmonar e capacidade funcional. Rev Bras Fisiot. 2010; 14(2): 91-8.

2. Cavalcante MCV, Lamy ZC, Filho FL, França AKTC, Santos AM, Thomaz, et al. Fatores associados à qualidade de vida de adultos em hemodiálise em uma cidade do Nordeste do Brasil. Jor Bras Nef. 2013; 35(2).

3. Nascimento LCA, Coutinho EB, Silva KNG. Efetividade do exercício físico na insuficiência renal crônica. Rev Fisiot Mov. 2012; 25(1): .231-239.

4. Dummer CD, Thomé FS, Veronese FV. Doença renal crônica, inflamação e aterosclerose: novos conceitos de um velho problema. Rev Assoc Méd Bras. 2007; 53(5).

5. Reboredo MM, Henrque DMN, Bastos MG, Paula RB. Exercício físico em pacientes dialisados. Rev Bras Med Esport. 2007, 13(6).
6. Terra, F.S.; Dias Costa, A.M.D.; Figueiredo, E.T.; Moraes, A.M.; Dias Costa, M.; Dias Costa, R. As principais complicações apresentadas pelos pacientes renais crônicos durante as sessões de hemodiálise. Rev Bras Clín Méd. 2010; 8(3):187-192.

7. Cuna MS, Andrade V, Guedes CAV, Meneghetti CHZ, Aguiar AP, Cardoso AL. Avaliação da capacidade funcional e da qualidade de vida em pacientes renais crônicos submetidos a tratamento hemodialítico. Rev Fisiot Pesq. 2009; 16(2).

8. Oller GASAO, Ribeiro RCHM, Travagim DSA, Batista MA, Marques S, Kusumota L. Functional independence in patients with chronic kidney disease being treated with haemodialysis. Rev Latino-Americ enf. 2012; 20(6):1033 - 1040.

9. Riberto M, Miyazaki MH, Juca SSH, Sakamoto H, Pinto PPN, Batistella LR. Validação da Versão Brasileira da Medida de Independência Funcional. Rev ACTA Fisiát. 2004; 11(2).

10. Peduto MDC. Acometimento da força e da funcionalidade dos membros superiores em pacientes com distrofia muscular de duchenne em corticoterapia. Dissertação (mestrado em Ciências) Faculdade de Medicina, Universidade de São Paulo, São Paulo, 2008, 101 p.

11. Ramos JS, Santos BM, Santos CA, Eumatsu ESC, Nagai JM, Bassini RF. Análise Comparativa dos Efeitos de um Programa de Exercícios para o Quadríceps em Pacientes durante a Hemodiálise. Rev Cient Index Link Jr. 2012; 2.

12. Bucuvic EM, Ponce D, Balbi AL. Fatores de risco para mortalidade na lesão renal aguda. Rev Assoc Méd Bras. 2011; 57(2):158-163.

13. Bortolotto LA. Hipertensão arterial e insuficiência renal crônica. Rev Bras Hipert. 2008; 15(3):152-155.

14. Lima FF, Miranda RCV, Rossi ES, Monteiro HL, Yen LS, Fahur B, et al. Avaliação Funcional Pré e pós-programa de exercício físico de pacientes em hemodiálise. Rev Med. 2013; 46(1):24-35.

15. Coelho CC, Aquino ES, Lara KL, Peres TM, Barja PR, Lima EM. Repercussões da insuficiência renal crônica na capacidade de exercício, estado nutricional, função pulmonar e musculatura respiratória de crianças e adolescentes. Rev Bras Fisiot. 2008; 12(1):1-8.

16. Corrêa LB, Oliveira RN, Cantareli F, Cunha LS. Efeito do treinamento muscular periférico na capacidade funcional e qualidade de vida nos pacientes em hemodiálise. Jor Bras Nef. 2009; 31(1): 18 - 24. 
17. Reis JS, Dantas MS, Silva CB, Valverde L, Landeiro RBR. Caracterização da Força Muscular Respiratória e da Capacidade Funcional de Pacientes Internados em uma Enfermaria. Rev Fisiot Saúd Func. 2012;. 1(2): 3 - 9.

18. Krug RR, Marchesan M, Krug MR, Moreira PR. Capacidade funcional e qualidade de vida de pacientes com insuficiência renal crônica. Rev Dig Buenos Aires. 2008; 121.

19. Mariano CM. Repercussões da doença renal crônica na postura, no equilíbrio e na capacidade funcional do indivíduo. Dissertação (Mestrado em Saúde Coletiva) Universidade de fortaleza. Fortaleza, 2013.

20. Grasselli CSM, Chaves ECL; Simão TP, Botelho PB, Silva RR. Avaliação da qualidade de vida dos pacientes submetidos à hemodiálise. Rev Socied Bras Clín Méd. 2012; 10(6).

21. Moura RMF, Silva FCR; Ribeiro GM, Sousa LAL. Efeitos do exercício físico durante a hemodiálise em indivíduos com insuficiência renal crônica: uma revisão. Rev Fisiot Pes. 2008; 15(1).

22. Soares KTA, Viesser MV, Rznisk TAB, Brum EP. Eficácia de um protocolo de exercícios físicos em pacientes com insuficiência renal crônica, durante o tratamento de hemodiálise, avaliada pelo SF-36. Rev Fisiot Mov. 2011; 24(1).
23. Costa PB, Vasconcelos KFS, Tassitano RM. Qualidade de vida: pacientes com insuficiência renal crônica no município de Caruaru, PE. Rev Fisiot Mov. 2010; 23(3):461 - 471.

24. Oller GASAO. Independência funcional e capacidade para o autocuidado de pacientes em tratamento hemodialítico. Dissertação (mestrado em Enfermagem) - Escola de Enfermagem de Ribeirão Preto, Universidade de São Paulo, Ribeirão Preto, 2012, 130 p.

25. Kovelis D, Pitta F, Probst VS, Peres CPA, Delfino VDA, Mocelin AJ, Brunetto AF. Função pulmonar e força muscular respiratória em pacientes com doença renal crônica submetidos à hemodiálise. Jor Bras Pneum. 2008; 34(11).

26. Barreto NDS. Recomendações e Aplicabilidade da Atividade Física no Paciente Renal Crônico em Tratamento Dialítico: Revisão. Rev Interfisio. 2013; 3(2).

27. Schneider J, Wendland J, Fassbinder TRc, Oliveira OB, Wilkemann ER. Resistência muscular localizada em portadores de insuficiência renal crônica que não realizam hemodiálise. Rev Cont Saúde. 2011; 10(20). 